\title{
Inertial and magnetic sensing of human movement near ferromagnetic materials
}

\author{
Daniel Roetenberg, Henk Luinge and Peter Veltink \\ University of Twente, The Netherlands \\ D.Roetenberg@utwente.nl
}

\begin{abstract}
This paper describes a Kalman filter design to estimate orientation of human body segments by fusing gyroscope, accelerometer and magnetometer signals. Ferromagnetic materials near the sensor disturb the local magnetic field and therefore the orientation estimation. The magnetic disturbance can be detected by looking at the total magnetic density and a magnetic disturbance vector can be calculated. Results show the capability of this filter to correct for magnetic disturbances.
\end{abstract}

\section{Introduction}

The use of small inertial sensors, like gyroscopes and accelerometers, has become common practice in motion tracking for mixed and augmented reality applications. Inertial sensors overcome problems like line-of-sight restrictions, limited range and latency related with other motion analysis systems. Typically, angular orientation of a body segment is determined by integrating the output from the angular rate sensors strapped on the segment. A relatively small offset error on the gyroscope signal will introduce large integration errors. Accelerometers measure the vector sum of acceleration a of the sensor and the gravitational acceleration $\mathbf{g}$. In most situations of human movement sensing, $\mathbf{g}$ is dominant, thus providing inclination information that can be used to correct the drifted orientation estimate from the gyroscopes. The principles for measuring orientation of a moving body segment fusing gyroscopes and accelerometers in a Kalman filter has been described by Luinge [3]. Results show accurate drift-free inclination estimation. The magnetometer is sensitive to the earth's magnetic field. It gives information about the heading direction in order to correct drift of the gyroscope about the vertical axis. Bachmann [1] and Foxlin [2] have implemented filters in which accelerometers and magnetometers are used for low frequency components of the orientation and gyroscopes to measure faster changes in orientation. These filters work in real-time and seem to be accurate and drift-free. However, ferromagnetic materials in the neighborhood of the sensor will disturb the local magnetic field and will therefore distort the orientation measurement. This interference impedes applications like ambulatory motion tracking. In this study, the Kalman filter by Luinge is extended with a magnetometer model that is able to compensate for magnetic disturbances.

\section{Kalman filter design}

A complementary Kalman filter was designed to estimate orientation, gyroscope offset and magnetic disturbance by combining 3D gyroscope, accelerometer and magnetometer signals using a model of the system and relevant signals (see Figure 1). Both accelerometers and gyroscopes make an estimate of the inclination $\mathbf{Z}$. The direction of the global magnetic vector $\mathbf{H}$ is estimated by the gyroscopes and magnetometers.

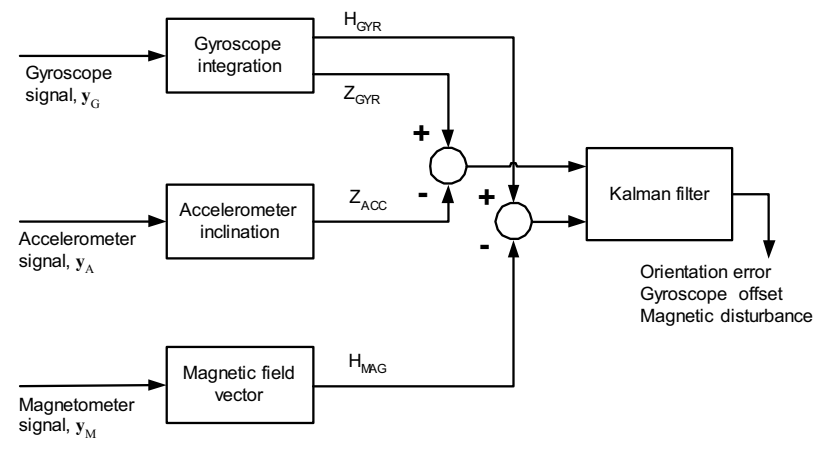

Figure 1: Kalman filter structure.

The magnetometer signal is described as the sum of the earth magnetic field $\mathbf{m}$, a disturbance vector $\mathbf{d}$ and a white noise term $\mathbf{v}_{\mathrm{M}}$ :

$$
\mathbf{y}_{M}=\mathbf{m}_{t}+\mathbf{d}_{t}+\mathbf{v}_{M, t}
$$

In a homogeneous magnetic field without metal, the disturbance $\mathbf{d}$ equals zero. The absolute value and the dip angle of the magnetic field are used as a measure of interference. If there is no disturbance, the total magnetic flux is constant. In the vicinity of metal, the magnetic flux and dip angle are most likely to change. In case of disturbance, less weight is assigned to the magnetometers and the estimation relies more on the gyroscopes and accelerometers. This period cannot be too long, since heading drift will be introduced. A disturbance vector $\mathbf{d}$ is calculated by taking the difference between the magnetic field vector derived by the gyroscopes and accelerometers and the direction of the actual magnetic field measured by the magnetometers. As long as the disturbance is within a specified boundary, the new magnetic field vector can be used as a reference. 


\section{Experiments}

For the experiments, a MT9 (Xsens) inertial and magnetic sensor module was used. To demonstrate the effect of magnetic disturbance on the filter, the sensor module was rotated manually + and -90 degrees along the three axes in metal free space and close to $3.75 \mathrm{~kg}$ of metal. Begin and end position are the same. The normalized magnetic field is presented in Figure 2. When no metal is near the sensor the field density is 1 . The disturbances are easily detectable (see arrows).

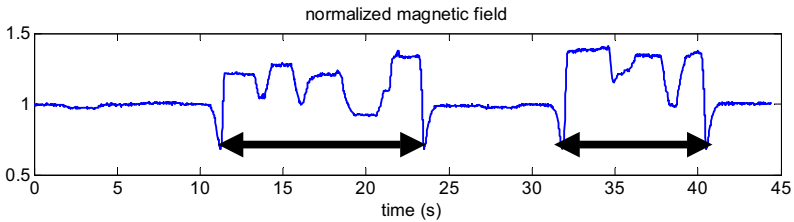

Figure 2: Normalized magnetic field with disturbances.

Figure 3 shows the Euler angles of rotation along the three axes when only gyroscopes are used. It can be seen that integration drift around the $\mathrm{x}$-axis is about 10 degrees after 45 seconds. Obviously, there is no magnetic disturbance noticeable.

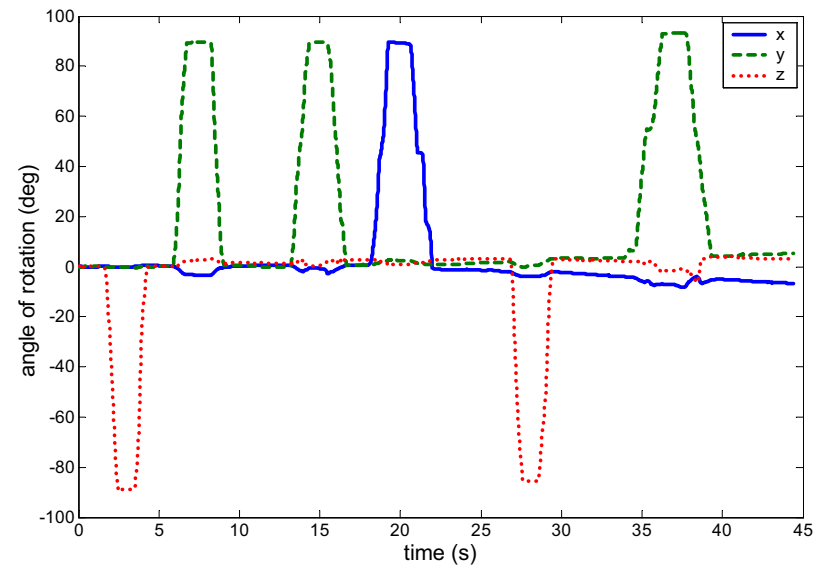

Figure 3: Angles of rotation using only gyroscopes.

Figure 4 shows the output when the Kalman filter weights the accelerometer and magnetic signals equally and no magnetic compensation is applied. From the start of the interference, the error becomes significant. Figure 5 illustrates the effect of the magnetic signal model on the Kalman filter. The disturbance vector $\mathbf{d}$ is calculated at the time instants where interference is detected. The output is drift-free and accurate.

\section{Conclusion and discussion}

Our Kalman filter implementation shows that accurate and drift-free orientation measurement near metal is possible when a magnetic disturbance model is used.

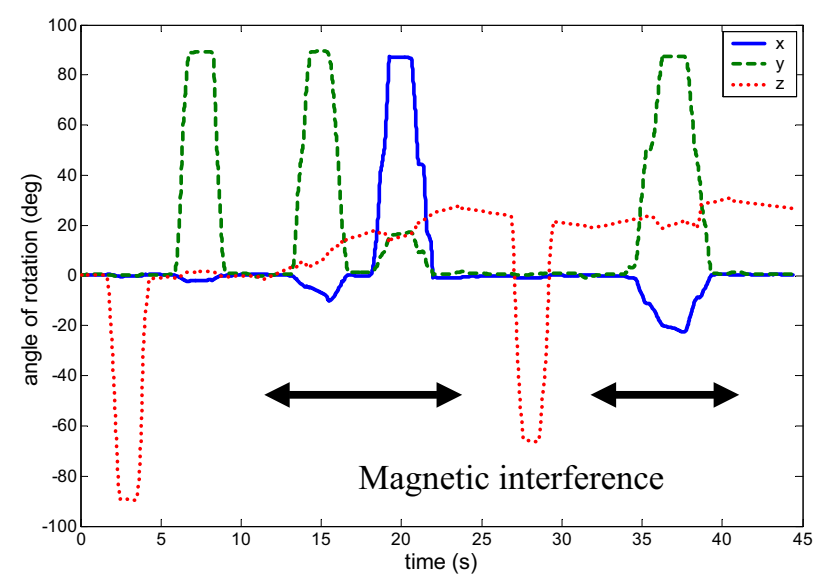

Figure 4: Angles of rotation with equal weight to accelerometers and magnetometers.

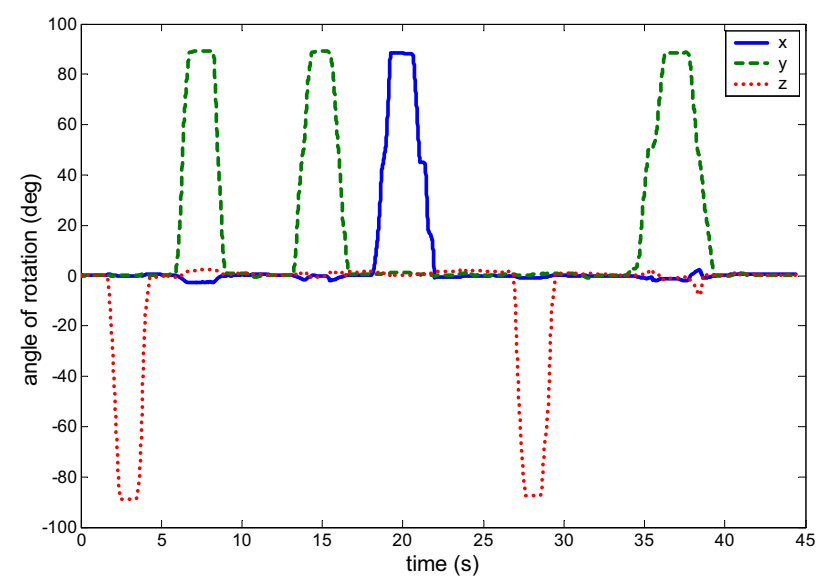

Figure 5: Angles of rotation with magnetic disturbance compensation.

In long trials, filter performance will decrease if disturbance is introduced slowly. The distinction between heading drift and disturbance is then difficult to make. The filter can be improved by considering other (redundant) signal characteristics; as for example, interference is more likely after a translation of the sensor, which can be measured by the accelerometers. The disturbance model is useful in many applications with ferromagnetic materials in the environment.

\section{References}

[1] Bachman, E.R., Inertial and magnetic tracking of limb segment orientation for inserting humans into synthetic environments. 2000: $\mathrm{PhD}$ Thesis.

[2] Foxlin, E., Inertial Head-Tracker Sensor Fusion by a Complementary Separate-Bias Kalman Filter. Proc. of VRAIS '96, 1996: p. 185-194.

[3] Luinge, H.J., Inertial sensing of human movement. 2002: $\mathrm{PhD}$ Thesis. 